chapters 3 to 8 the flow problem is first described before the associated forced convection problem - which arises when the drop, bubble or particle has a concentration not equal to that of the continuous phase-is discussed. This systematic plan enables the reader easily to find his way about in individual chapters.

The last four chapters cover wall effects, shear and accelerated flow and formation and break-up of fluid particles, necessarily rather briefly.

This reviewer has no doubt of the

\section{Ionosphere phenomena}

Non-Linear Phenomena in the Ionosphere. By A. V. Gurevich. (Springer: New York, Heidelberg and Berlin, 1978.) DM98; \$49.

This monograph is the tenth in the series published by Springer under the general title Physics and Chemistry in Space, which is intended "to provide student teachers and researchers with clear and concise presentations of upto-date topics in space phenomena". Each of the volumes is prepared by a recognised international authority in the field, and this latest text is a very worthy addition to the series.

In April 1933, during the early years of ground-based radio wave studies of the ionosphere, Tellegen observed that radio signals received at Eindhoven, Holland, on 460 metres from the Swiss station Beromünster were modulated by signals from the newly established high-power Luxembourg station operating on 1,190 metres. It was soon established that the phenomenon was not due to cross-modulation within the receiving equipment, and in 1934 V. A. Bailey and D. F. Martyn showed theoretically how this so-called 'Luxembourg effect' could be explained in terms of non-linear interaction of the two radio signals in the lower part of the ionosphere. In the years immediately following World War II the theory of the phenomena became more fully worked out and wave-interaction was developed as an additional diagnostic tool for studying the lower levels of the ionosphere. In this period cross-modulation resonance effects of gyrofrequencies were also observed, and the possibility of ionospheric 'self-demodulation' discussed theoretically and observed experimentally. In recent years the availability of radio transmitters radiating power large enough to perturb markedly the lower ionosphere and to modify the ionosphere at higher levels, has resulted in a greatly renewed interest by both theoreticians and experimentalists in many other aspects of ionospheric wave interaction pheno- value of the book to chemical engineers and others with a special interest in drops, bubbles and particles. However, it is to be hoped that it will persuade theoretical hydrodynamicists to take an interest in some of the fascinating unsolved problems, particularly in the 'skirted' and 'dimpled' cap bubbles described in chapter 8.

D. W. Moore

D. W. Moore is Professor of Applied Mathematics at Imperial College, University of London, UK.

mcna. The variety of ionospheric modifications successfully effected is now quite extensive and includes large-scale stratifications leading to the creation of an artificial 'sporadic F' layer, the formation of inhomogeneities elongated along the magnetic field, the excitation of plasma and ion-acoustic waves, luminescence produced by accelerated electrons (a possibility first considered

\section{Group theory and nuclear symmetries}

Group Symmetries in Nuclear Structure. By J. C. Parikh. Pp. 277. (Plenum: New York and London, 1978.) \$40.20.

THE structure of the atomic nucleus presents a demanding and complex problem to the physicist, with ramifications which touch on many areas of fundamental research. As with atomic structure, the source of the complexity is that it is a problem with many particles in interaction, but not so very many that the change from $n$ particles to $n+1$ is not significant-indeed, those changes are amongst the most interesting features of the problem. But in atomic physics one has the advantage that the fundamental interaction between the particles involved is known and simple; it is the electromagnetic interaction. The nuclear structure physicist must cope with the complicated, largely empirically determined form of the nuclear forces. Of enormous value in bringing order to this complexity is the use of group theory to exploit the symmetries, exact and approximate, fundamental and phenomenological, which nuclei possess.

This monograph is concerned with presenting these symmetries, especially the dynamical symmetries which arise in the finite-dimensional state-space of approximation schemes like the shell model, and at the same time with the introduction of the basic mathematical ideas and techniques of group theory. by V. A. Bailey in 1937), and the precipitation of fast electrons from the plasmasphere.

For more than two decades major contributions to the theory of these various non-linear phenomena have been made by Dr A. V. Gurevich, and the clarity and conciseness of this volume is a testimony to his profound understanding of the subject.

Apart from one or two very minor matters, the translation is excellent and the standard of printing and production of the volume are both of the very highest quality. The monograph is well indexed, includes a comprehensive list of references, and can be strongly recommended to all concerned with this very topical aspect of ionosplteric physics.

W. J. Granville Beynon

Sir Granville Beynon is Professor and Head of the Department of Physics at the University College of Wales, Aberystwyth, $U K$.

The level of the treatment is appropriate to postgraduate students and research workers. It is intended to be useful to both experimentalists and theorists, but I doubt if any but the most dedicated experimentalists would in fact wish to tackle the book in its entirety. It is a specialised monograph, a review rather than a textbook. To my mind it would have been more useful if it had placed the specific topics covered in a broader context. The nuclear physics is used to illustrate methods and problems in group theory rather than the other way round, and in my view that is a weakness. However, there are good introductions to the important topics of seniority and symplectic symmetry, of Wigner's SU(4) and Elliott's SU(3), and of their extensions. An interesting treatment of a method due to French for deriving good approximations to binding energies, spectra, and so on, by using moments of the spectral distribution of the Hamiltonian, forms a central feature of the monograph.

For the nuclear structure theorist who wants a detailed study of the use and application of group theory in this field, I know of no other recent book. Although the work originated in a lecture course given in 1970, some more recent work is cited; it's a pity, however, that the latest reference brings us no further than to 1975 .

In summary, this is a competent but specialist treatment for the specialist worker in the field.

John M. Charap

John M. Charap is Professor cf Theoretical Physics at Queen Mary College, University of London, UK. 